\title{
UNIFORMLY FAT SETS
}

\author{
JOHN L. LEWIS
}

\begin{abstract}
In this paper we study closed sets $E$ which are "locally uniformly fat" with respect to a certain nonlinear Riesz capacity. We show that $E$ is actually "locally uniformly fat" with respect to a weaker Riesz capacity. Two applications of this result are given. The first application is concerned with proving Sobolev-type inequalities in domains whose complements are uniformly fat. The second application is concerned with the Fekete points of $E$.
\end{abstract}

Introduction. Let $x=\left(x_{1}, x_{2}, \ldots, x_{n}\right)$ be a point in Euclidean $n$ space, $\mathbf{R}^{n}$, with $|x|$ the norm of $x$. For $\alpha>0$, let $I_{\alpha}(x)=|x|^{(\alpha-n)}$ denote the Riesz kernel of order $\alpha$ and put

$$
\begin{array}{ll}
I_{\alpha} * f(x)=\int_{\mathbf{R}^{n}}|x-y|^{(\alpha-n)} f(y) d y, & x \in \mathbf{R}^{n}, \\
I_{\alpha} * \mu(x)=\int_{\mathbf{R}^{n}}|x-y|^{(\alpha-n)} d \mu(y), & x \in \mathbf{R}^{n},
\end{array}
$$

when $f$ is a Lebesgue integrable function and $\mu$ a Borel measure on $\mathbf{R}^{n}$. Here $d y$ denotes Lebesgue measure on $\mathbf{R}^{n}$. If $E \subseteq \mathbf{R}^{n}, 0<\alpha p<n$ and $p>1$, define the $(\alpha, p)$ outer Riesz capacity of $E$ by

$$
R_{\alpha, p}(E)=\inf \left\{\|f\|_{p}^{p}: I_{\alpha} * f \geq 1 \text { on } E\right\}
$$

where $\|f\|_{p}$ is the Lebesgue $p$ norm of $f$. We note that $\alpha=1, p=2$, is the classical Newtonian capacity. If $\alpha p=n$, we let

$$
R_{\alpha, p}(E)=\inf \left\{\|f\|_{p}^{p}: J_{\alpha} * f \geq 1 \text { on } E\right\}
$$

where $J_{\alpha}$ is the truncated Riesz kernel defined by

$$
\begin{aligned}
J_{\alpha}(x) & =|x|^{(\alpha-n)}-(100)^{(\alpha-n)}, \quad|x| \leq 100, \\
& =0, \quad|x|>100 .
\end{aligned}
$$

We shall say that a property holds $(\alpha, p)$ quasi everywhere (abbreviated $(\alpha, p)$ q.e.) on a set $E$ if it holds on $E$ except perhaps for a set of $R_{\alpha, p}$ capacity zero. Next we list some properties of Riesz capacities. To simplify the writing we state these properties only for $0<\alpha p<n$. However (A)-(C) also hold when $\alpha p=n$ provided $I_{\alpha}$ is replaced by $J_{\alpha}$. If $E$ is a compact set, then

(A) $R_{\alpha, p}(E)=\sup \left\{\nu(E)^{p}: \operatorname{supp} \nu \subseteq E\right.$ and $\left.\left\|I_{\alpha} * \nu\right\|_{p^{\prime}} \leq 1\right\}$, for $p^{\prime}=p /(p-1)$,

(B) $R_{\alpha, p}(E)=\sup \left\{\nu(E): I_{\alpha} *\left(I_{\alpha} * \nu\right)^{1 /(p-1)} \leq 1\right.$ on $\left.\operatorname{supp} \nu \subseteq E\right\}$,

(C) there exists a measure $\mu$ for which $P=I_{\alpha} *\left(I_{\alpha} * \mu\right)^{1 /(p-1)} \geq 1,(\alpha, p)$ q.e., on $E$ and $P=1,(\alpha, p)$ q.e., on $\operatorname{supp} \mu \subseteq E$. Moreover,

$$
\mu(E)=\left\|I_{\alpha} * \mu\right\|_{p^{\prime}}^{p^{\prime}}=R_{\alpha, p}(E) .
$$

Received by the editors March 11, 1987.

1980 Mathematics Subject Classification (1985 Revision). Primary 31C15, 31B15. 
(D) If $B(x, r)=\{y:|y-x|<r\}$, then $R_{\alpha, p}[B(x, r)] \sim r^{(n-\alpha p)}$, where $\sim$ means the ratio of the two functions is bounded above and below by a positive constant,

(E) If $H^{m}$ denotes $m$-dimensional Hausdorff measure on $\mathbf{R}^{n}$, then

(i) $H^{(n-\alpha p)}(E)<\infty \rightarrow R_{\alpha, p}(E)=0$,

(ii) $H^{(n-\alpha p+\varepsilon)}(E)>0 \rightarrow R_{\alpha, p}(E)>0, \varepsilon>0$,

(F) If $\beta>0, q>1$, and either $\beta q<\alpha p$ or $\beta q=\alpha p$ and $\beta<\alpha$, then

$$
R_{\beta, q}(E)^{(n-\alpha p)} \leq A R_{\alpha, p}(E)^{(n-\beta q)},
$$

where $A$ is a positive constant independent of $E$.

Note from $(\mathrm{F})$ that $R_{\alpha, p}$ is a stronger capacity than $R_{\beta, q}$ in the sense that $R_{\alpha, p}(E)=0 \rightarrow R_{\beta, q}(E)=0$. For the proof of (A), (C), (D) and (E) see [14]. (B) is given in $[2]$ and $(\mathrm{F})$ can be found in $[\mathbf{3}]$. Finally we mention that a general survey of nonlinear potential theory as well as an outline of the results in this paper can be found in $[\mathbf{1 3}]$.

In classical potential theory a set can be defined to be fat at a point in several equivalent ways. The most natural definition for nonlinear potential theory turns out to be: A set $E$ is said to be $(\alpha, p)$ fat at $x$ for $0<\alpha p<n$ if

$$
\int_{0}^{1}\left[r^{(\alpha p-n)} R_{\alpha, p}(E \cap B(x, r))\right]^{1 /(p-1)} \frac{d r}{r}=+\infty .
$$

Otherwise $E$ is said to be $(\alpha, p)$ thin at $x$.

A similar definition holds for $\alpha p=n$. We shall say that a set $E$ is $(\alpha, p)$ locally uniformly fat for $0<\alpha p \leq n, p>1$, provided there exist positive constants $r_{0}$ and $\lambda$ such that

$$
R_{\alpha, p}\left(r^{-1}[B(x, r) \cap E]\right) \geq \lambda
$$

for every $x$ in $E$ and $0<r<r_{0}$. Here

$$
r^{-1}[B(x, r) \cap E]=\left\{x+r^{-1}(y-x): y \in B(x, r) \cap E\right\} .
$$

If $0<\alpha p<n$ we note that (1.1) is equivalent to

$$
r^{(\alpha p-n)} R_{\alpha, p}[B(x, r) \cap E] \geq \lambda
$$

for every $x$ in $E$ and $0<r<r_{0}$, as follows from the dilation properties of Riesz capacities.

If (1.1) holds for $0<r<\infty$ and every $x$ in $E$ we say that $E$ is uniformly fat. In the classical case $\alpha=1, p=2$, domains whose complements satisfy (1.1) have been considered in several recent papers (see $[6,10,18]$ ).

We note from $(\mathrm{F})$ that a $(\beta, q)$ locally uniformly fat set is also $(\alpha, p)$ uniformly fat when either $\beta q<\alpha p<n$ or $\alpha p=\beta q<n$ and $\alpha>\beta$. In this paper we show that if a closed set $E$ is locally uniformly fat with respect to a given capacity, then $E$ is also locally uniformly fat with respect to a weaker capacity and corresponding Hausdorff measure. More specifically, we prove

THEOREM 1. Given $0<\alpha p \leq n, p>1$, suppose $E$ is closed and $(\alpha, p)$ locally uniformly fat. Then there exists $\varepsilon, \lambda_{1}>0$ depending only on $\alpha, p, n, \lambda$, such that whenever $x \in E, 0<r<r_{0}$, and $\alpha p-\varepsilon<\beta q<\alpha p$, we have

$$
\begin{gathered}
R_{\beta, q}[E \cap B(x, r)] \geq \lambda_{1} r^{(n-\beta q)}, \\
H^{(n-\alpha p+\varepsilon)}[E \cap B(x, r)] \geq \lambda_{1} r^{(n-\alpha p+\varepsilon)} .
\end{gathered}
$$


We give two applications of Theorem 1. In order to state the first application we need some notation. Let $D$ be an open set, $C_{0}^{\infty}(D)$ the space of infinitely differentiable functions with compact support in $D$, and $d(x)$ the distance from $x$ to $\mathbf{R}^{n}-D$. In $\S 5$ we prove

THEOREM 2. Let $D$ be an open set and $1<p \leq n$. If $\mathbf{R}^{n}-D$ is $(1, p)$ uniformly fat, then

$$
\int_{D}\left[\frac{u(x)}{d(x)}\right]^{p} d x \leq A \int_{D}|\nabla u|^{p} d x
$$

for each $u \in C_{0}^{\infty}(D)$. Here $A>0$ depends only on $p, \lambda$, and $n$.

In Theorem 2, $\nabla u$ denotes the gradient of $u$. We note that Ancona [6] proved Theorem 2 when $p=2$ and showed that it is best possible for $n=2, p=2$. In $\S 5$ we also point out that (1.5) holds for $p>n$ whenever $D \neq \mathbf{R}^{n}$. In $\S 6$ we examine the extent to which (1.5) implies $\mathbf{R}^{n}-D$ is uniformly fat when $1<p \leq n$. In case $D=B(0,1)-\{0\}$ and $1<p<n$, it can be shown using Hardy's inequality on rays that (1.5) holds for some fixed $A>0$. Clearly $\mathbf{R}^{n}-D$ is not uniformly fat. Thus (1.5) is not sufficient for $\mathbf{R}^{n}-D$ to be uniformly fat when $1<p<n$. However we can prove

THEOREM 3. If $p=n$ and (1.5) holds for some fixed $A>0$, then $\mathbf{R}^{n}-D$ is uniformly fat. Moreover, there exists a compact set $F \subseteq B(0,1)$ with $H^{\varepsilon}(F)=0$ for each $\varepsilon>0$ with the following property: If $D=B(0,1)-F$ and $A, p, 1<p<n$, are given positive numbers, then (1.5) fails to hold for some $u$ in $C_{0}^{\infty}(D)$.

Next let $E \subseteq B(0,1)$ be a compact set, $m$ a given positive integer, and $0<\alpha<$ $n / 2$. Recall that a sequence $\left(x_{i}\right)_{1}^{m}$ of $m$ points in $E$ which minimizes

$$
\sum_{i \neq j}\left|y_{i}-y_{j}\right|^{(2 \alpha-n)}
$$

over all sequences $\left(y_{i}\right)_{1}^{m}$ of $m$ points in $E$, is called a sequence of $m$ Fekete points of $E$ corresponding to the Riesz kernel $I_{2 \alpha}$. In $\S 7$ we point out that Theorem 1 implies

THEOREM 4. Let $E$ and $\left(x_{i}\right)_{1}^{m}$ be as above for fixed $\alpha, 0<\alpha<n / 2$, and suppose that $E$ is $(\alpha, 2)$ locally uniformly fat. Then there exists $\varepsilon, a>0$ such that

$$
\min _{i \neq j}\left|x_{i}-x_{j}\right| \geq a(1 / m)^{(n-2 \alpha+\varepsilon)}
$$

where $a$ and $\varepsilon$ depend only on $\alpha, n, \lambda$, and $r_{0}$.

We note that related theorems have been proved in $[8,16$, and 17]. In $\S 7$ we also indicate that Theorem 4 has an analogue for $\alpha=n / 2$.

Finally in this section we would like to thank Professor D. R. Adams for many useful conversations regarding Riesz capacities.

2. Proof of Theorem 1 for $\alpha \geq 2$. In the sequel $c$ denotes a positive constant which may only depend on $\alpha, p$, and $n$, not necessarily the same at each occurrence. Also $\bar{A}$ and $\partial A$ denote the closure and boundary of the set $A$. We now begin the proof of Theorem 1. Fix $R, 0<R \leq r_{0}$, and suppose $E$ is $(\alpha, p)$ locally uniformly 
fat. Given $x_{0} \in E$, we claim there exists a compact set $F$ contained in $\bar{B}\left(x_{0}, R\right) \cap E$ with

$$
R_{\alpha, p}\left(r^{-1}[F \cap B(x, r)]\right) \geq c \lambda,
$$

for $0<r \leq R$ and every $x \in F$. The set in parentheses is defined as in $\S 1$. Thus $F$ is $(\alpha, p)$ locally uniformly fat. To construct $F$ let $E_{1}=E \cap B\left(x_{0}, R / 2\right)$ and inductively let

$$
E_{m}=\left[\bigcup_{x \in E_{m-1}} B\left(x, 2^{-m} R\right)\right] \cap E, \quad m=2,3, \ldots
$$

Then if suffices to let $F$ be the closure of $\bigcup_{m=1}^{\infty} E_{m}$, as is easily shown using (1.1).

The proof of Theorem 1 will be divided into three parts. In $\S 3$ we prove Theorem 1 for $0<\alpha<2, \alpha p<n$. In $\S 4$ the proof of Theorem 1 for $\alpha p=n$ is given. In this section we prove Theorem 1 for $\alpha \geq 2$ and $\alpha p<n$. To this end let $P=I_{\alpha} *\left(I_{\alpha} * \mu\right)^{1 /(p-1)}$ be the equilibrium potential for $F$ (see (C) of $\S 1$ ). We first establish certain Wiener-type estimates on $P$ which will in fact imply that $P$ is Hölder continuous on $\mathbf{R}^{n}$. Using the relationship between $P$ and $\mu$ it is then relatively easy to prove (1.3) and (1.4). To establish these estimates we shall need some notation. Let $u=1-P$ and for fixed $x_{1} \in \operatorname{supp} \mu$ with $P\left(x_{1}\right)=1$ let

$$
M(t)=M\left(t, u, x_{1}\right)=\sup _{x \in B\left(x_{1}, t\right)} u(x), \quad t>0 .
$$

If $\alpha \geq 2$, note that since $\alpha p<n$, and $p>1$, we must have $n \geq 3$. Also in this case $I_{\alpha}$ and consequently $P$ are superharmonic in $\mathbf{R}^{n}$. Thus $u=1-P$ is subharmonic and we can write $u$ in $B\left(x_{1}, s\right), s>0$, in the form $u=h-q$, where $h$ is the least harmonic majorant of $u$ in $B\left(x_{1}, s\right)$ and $q$ is a Green's potential in $B\left(x_{1}, s\right)$. $h$ can be written explicitly in terms of a Poisson integral as

$$
h(x)=c s^{-1} \int_{\partial B\left(x_{1}, s\right)} \frac{\left(s^{2}-\left|x_{1}-x\right|^{2}\right)}{|y-x|^{n}} u(y) d H^{n-1} y, \quad x \in B\left(x_{1}, s\right) .
$$

For fixed $s \leq R$ and $0<s_{1} \leq \frac{1}{2} s$ we first show that if

$$
\left|h(x)-h\left(x_{1}\right)\right| \leq \frac{1}{4} M(s, h), \quad x \in B\left(x_{1}, s_{1}\right),
$$

then there exists $\beta, \frac{3}{4} \leq \beta<1$, depending only on $\alpha, p, n$, and $\lambda$ such that

$$
M\left(\frac{1}{8} s_{1}, u\right) \leq \beta M(s, h) .
$$

Here, $M(s, h)=\sup _{x \in B\left(x_{1}, s\right)} h(x)$.

To prove (2.4) under assumption (2.3) note that either $h\left(x_{1}\right) \geq \frac{1}{2} M(s, h)$ or (2.4) holds with $\beta=\frac{3}{4}$ since $u \leq h$ in $B\left(x_{1}, s\right)$. If $h\left(x_{1}\right) \geq M(s, h) / 2$, then from (2.3), (C) of $\S 1$, and the definition of $u, q, h$, we deduce

$$
q(x) \geq h(x) \geq M(s, h) / 4, \quad(\alpha, p) \text { q.e. on } F \cap B\left(x_{1}, s_{1}\right) .
$$

Next we note for $\alpha>2$ that

$$
\Delta u=-\Delta P=c I_{(\alpha-2)} *\left(I_{\alpha} * \mu\right)^{1 /(p-1)}
$$

for $H^{r_{n}}$ almost every $x$ in $\mathbf{R}^{n}$, while $\Delta u=c\left(I_{2} * \mu\right)^{1 /(p-1)}$ if $\alpha=2$. If $\alpha>2$ and

$$
g(x, y)=|x-y|^{(2-n)}-\left|\left(x-x_{1}\right)\right| y-\left.x_{1}\right|^{2}-\left.\left(y-x_{1}\right) s^{2}\right|^{(2-n)}\left(s\left|y-x_{1}\right|\right)^{(n-2)},
$$


$x, y \in B\left(x_{1}, s\right)$, denotes the Green's function for $B\left(x_{1}, s\right)$ with pole at $y \in B\left(x_{1}, s\right)$, it follows that

$$
\begin{aligned}
q(x) & =c \int_{B\left(x_{1}, s\right)} g(x, y)\left[I_{(\alpha-2)} *\left(I_{\alpha} * \mu\right)^{1 /(p-1)}\right](y) d y \\
& =c \int_{B\left(x_{1}, s_{1} / 2\right)} \cdots+c \int_{B\left(x_{1}, s\right)-B\left(x_{1}, s_{1} / 2\right)} \cdots \\
& =K_{1}(x)+K_{2}(x)
\end{aligned}
$$

If $\alpha=2$ replace the first term in brackets by the expression for $\Delta u$. Observe that $K_{2}$ is a positive harmonic function in $B\left(x_{1}, s_{1} / 2\right)$. Hence by Harnack's inequality either $K_{2}<\frac{1}{8} M(s, h)$ at each point of $B\left(x_{1}, s_{1} / 4\right)$ or $K_{2} \geq c M(s, h)$ at each point of $B\left(x_{1}, \frac{1}{4} s_{1}\right)$. If the second possibility occurs it follows easily from $u \leq h$ and $q \geq K_{2}$ that (2.4) is valid. If the first possibility occurs, then from (2.5) we deduce that $K_{1} \geq \frac{1}{8} M(s, h),(\alpha, p)$ q.e. on $F \cap B\left(x_{1}, \frac{1}{4} s_{1}\right)$.

We continue under the above assumption. Observe for $x \in B\left(x_{1}, s_{1} / 4\right), y \in$ $B\left(x_{1}, s_{1} / 2\right)$, that there exists a positive constant $c$ with

$$
|x-y|^{(2-n)} \leq c\left[|x-y|^{(2-n)}-(s / 2)^{(2-n)}\right] \leq c g(x, y),
$$

where the last inequality follows easily from the maximum principle for harmonic functions. Also

$$
g(x, y) \leq|x-y|^{(2-n)}, \quad x, y \in B\left(x_{1}, s\right) .
$$

Let $\mu_{1}=\mu / B\left(x_{1}, s_{1} / 4\right)$ and $\mu_{2}=\mu-\mu_{1}$. Using (2.7) we get for $x \in B\left(x_{1}, s_{1} / 4\right)$ and $\alpha>2$

$$
\begin{aligned}
K_{1}(x)= & c \int_{B\left(x_{1}, s_{1} / 2\right)} g(x, y)\left[I_{(\alpha-2)} *\left(I_{\alpha} * \mu\right)^{1 /(p-1)}\right](y) d y \\
\leq & c \int_{B\left(x_{1}, s_{1} / 2\right)}|x-y|^{(2-n)}\left[I_{\alpha-2} *\left(I_{\alpha} * \mu_{1}\right)^{1 /(p-1)}\right](y) d y \\
& +c \int_{B\left(x_{1}, s_{1} / 2\right)}|x-y|^{(2-n)}\left[I_{\alpha-2} *\left(I_{\alpha} * \mu_{2}\right)^{1 /(p-1)}\right](y) d y \\
= & L_{1}(x)+L_{2}(x) .
\end{aligned}
$$

If $\alpha=2$ replace the last two terms in brackets by the expression for $\Delta u$.

We claim there exists $c>0$ such that

$$
L_{2}(z) \leq c L_{2}(w), \quad z, w \in B\left(x_{1}, s_{1} / 8\right) .
$$

To prove this claim use the fact that

$$
I_{\alpha} * \mu_{2}(z) \leq c I_{\alpha} * \mu_{2}(w), \quad z, w \in B\left(x_{1}, 3 s_{1} / 16\right),
$$

(since supp $\mu_{2} \subseteq \mathbf{R}^{n}-B\left(x_{1}, s_{1} / 4\right)$ ) to deduce that

$$
I_{(\alpha-2)} *\left(I_{\alpha} * \mu_{2}\right)^{1 /(p-1)}(z) \leq c\left[I_{(\alpha-2)} *\left(I_{\alpha} * \mu_{2}\right)^{1 /(p-1)}\right](w)
$$

for $z, w \in B\left(x_{1}, 5 s_{1} / 32\right)$ and $\alpha>2$. Next write $L_{2}$ as a sum of integrals over $B\left(x_{1}, 5 s_{1} / 32\right)$ and $B\left(x_{1}, s_{1} / 2\right)-B\left(x_{1}, 5 s_{1} / 32\right)$, and use the above inequality. There are obvious modifications if $\alpha=2$. We omit the details. Using (2.8) we find that 
either $L_{2} \leq \frac{1}{16} M(s, h)$ at every point of $B\left(x_{1}, s_{1} / 8\right)$ or $L_{2} \geq c M(s, h)$ at each point of $B\left(x_{1}, s_{1} / 8\right)$. If the second alternative occurs, then from (2.6) we deduce that

$$
q \geq K_{1} \geq c L_{2} \geq c M(s, h)
$$

on $B\left(x_{1}, s_{1} / 8\right)$, from which (2.4) follows as previously. If the first alternative occurs, then

$$
L_{1}(x) \geq\left(K_{1}-L_{2}\right)(x) \geq M(s, h) / 16, \quad(\alpha, p) \text { q.e., on } F \cap B\left(x_{1}, s_{1} / 8\right) .
$$

In this case since $I_{a} * I_{b}$ is a constant multiple of $I_{a+b}$ when $a, b>0$ and $0<$ $a+b<n$, we have

$$
L_{1} \leq c I_{\alpha} *\left(I_{\alpha} * \mu_{1}\right)^{1 /(p-1)}
$$

It follows from this inequality, (2.9) and the definition of $R_{\alpha, p}$ capacity that

$$
\begin{aligned}
M(s, h)^{p} R_{\alpha, p}\left[B\left(x_{1}, \frac{s_{1}}{8}\right) \cap F\right] \leq c \int_{\mathbf{R}^{n}}\left(I_{\alpha} * \mu_{1}\right)^{p /(p-1)}(x) d x \\
=c \int_{\mathbf{R}^{n}}\left(I_{\alpha} * \mu_{1}\right)^{1 /(p-1)}(x)\left(I_{\alpha} * \mu_{1}\right)(x) d x \\
=c \int_{\mathbf{R}^{n}} I_{\alpha} *\left(I_{\alpha} * \mu_{1}\right)^{1 /(p-1)} d \mu_{1} .
\end{aligned}
$$

To proceed further we need the inequality

$$
\left[s_{1}^{(\alpha p-n)} \mu_{1}\left(\mathbf{R}^{n}\right)\right]^{1 /(p-1)} \leq c\left[I_{\alpha} *\left(I_{\alpha} * \mu_{1}\right)^{1 /(p-1)}\right] \leq c K_{1},
$$

for each $x \in B\left(x_{1}, s_{1} / 4\right)$. The left inequality follows easily from the fact that supp $\mu_{1} \subseteq B\left(x_{1}, s_{1} / 4\right)$. To prove the right inequality let $\chi$ be the characteristic function of $B\left(x_{1}, s_{1} / 2\right)$ and note from (2.6) that for $\alpha>2$

$$
\begin{aligned}
K_{1}(x) & \geq c \int_{\mathbf{R}^{n}} \chi(y)|x-y|^{(2-n)}\left[I_{(\alpha-2)} *\left(I_{\alpha} * \mu_{1}\right)\right]^{1 /(p-1)}(y) d y \\
& =c \int_{\mathbf{R}^{n}}\left[\int_{\mathbf{R}^{n}} \chi(y)|x-y|^{(2-n)}|y-z|^{(\alpha-2-n)} d y\right]\left(I_{\alpha} * \mu_{1}\right)^{1 /(p-1)}(z) d z \\
& =\int_{\mathbf{R}^{n}} T(x, z)\left(I_{\alpha} * \mu_{1}\right)^{1 /(p-1)}(z) d z
\end{aligned}
$$

when $x \in B\left(x_{1}, s_{1} / 4\right)$, where we have used the Tonelli Theorem to interchange the order of integration in the second integral. If $z \in B\left(x_{1}, s_{1}\right), x \in B\left(x_{1}, s_{1} / 4\right)$, and $|x-z| \geq \frac{1}{8} s_{1}$, then clearly

$$
T(x, z) \geq c s_{1}^{(\alpha-n)} \geq c|x-z|^{(\alpha-n)} .
$$

Otherwise if $A=\left\{y \in B\left(x_{1}, s_{1} / 2\right): 2|x-z|<|y-x|\right\}$, then from the geometry of the situation we see for $x \in B\left(x_{1}, s_{1} / 4\right)$

$$
T(x, z) \geq c \int_{A}|x-y|^{(\alpha-n)} d y \geq c|x-z|^{(\alpha-n)} .
$$

From (2.12) it follows in either case that

$$
K_{1}(x) \geq c \int_{B\left(x_{1}, s_{1} / 2\right)}|x-z|^{(\alpha-n)}\left(I_{\alpha} * \mu_{1}\right)^{1 /(p-1)}(z) d z=S(x)
$$


for each $x$ in $B\left(x_{1}, s_{1} / 4\right)$. Also $I_{\alpha} * \mu_{1}(z) \leq c \mu_{1}\left(\mathbf{R}^{n}\right)\left|x_{1}-z\right|^{(\alpha-n)}$, for $z$ in $\mathbf{R}^{n}-$ $B\left(x_{1}, s_{1} / 2\right)$. Using this inequality and (2.13) it follows for $x \in B\left(x_{1}, s_{1} / 4\right)$ and $\alpha>2$ that

$$
\begin{aligned}
I_{\alpha} *\left(I_{\alpha} * \mu_{1}\right)^{1 /(p-1)}(x) & \leq c S(x)+c\left[s_{1}^{(\alpha p-n)} \mu_{1}\left(\mathbf{R}^{n}\right)\right]^{1 /(p-1)} \\
& \leq c S(x) \leq c K_{1}(x) .
\end{aligned}
$$

If $\alpha=2$, the above inequality follows directly from (2.6). Thus (2.11) is true. Using the right-hand inequality of (2.11) in (2.10) and the fact (see (C) of $\S 1$ ) that

$$
K_{1}(x) \leq q(x)=h(x) \leq M(s, h),
$$

$(\alpha, p)$ q.e. on $\operatorname{supp} \mu_{1}$, we obtain

$$
M(s, h)^{p} R_{\alpha, p}\left[B\left(x_{1}, s_{1} / 8\right) \cap F\right] \leq c M(s, h) \mu_{1}\left(\mathbf{R}^{n}\right) .
$$

Using the left inequality in (2.11) it follows that

$$
M(s, h)\left\{s_{1}^{(\alpha p-n)} R_{\alpha, p}\left[B\left(x_{1}, s_{1} / 8\right) \cap F\right]\right\}^{1 /(p-1)} \leq c K_{1} \leq c q
$$

for each $x$ in $B\left(x_{1}, s_{1} / 4\right)$. The left-hand side of this inequality is bounded below by $c \lambda^{1 /(p-1)} M(s, h)$ since $F$ is locally uniformly fat. Hence, if $x \in B\left(x_{1}, s_{1} / 8\right)$, then

$$
u(x) \leq h(x)-q(x) \leq\left(1-c \lambda^{1 /(p-1)}\right) M(s, h),
$$

so (2.4) is valid when $\alpha \geq 2$ and $0<\alpha p<n$.

Next observe from (2.2) that for $x \in B\left(x_{1}, s / 2\right)$, and $u^{+}=\max (u, 0)$,

$$
\begin{aligned}
|\nabla h|(x) & \leq c s^{-n} \int_{\partial B\left(x_{1}, s\right)}|u(y)| d H^{n-1} y \leq c s^{-n} \int_{\partial B\left(x_{1}, s\right)} u^{+}(y) d H^{n-1} y \\
& \leq c s^{-1} M(s, u)=c s^{-1} M(s, h),
\end{aligned}
$$

where we have used $u\left(x_{1}\right)=0$ and the sub mean value property of subharmonic functions. Hence there exist $\delta>0$ such that if $s_{1}=8 \delta s$, then (2.3) and consequently (2.4) hold.

To continue the proof of Theorem 1, we now iterate (2.4) starting with $r_{0}=R$ and continuing with $r_{k}=\delta^{k} R, k=1,2 \ldots$ Applying (2.4) with $s=r_{i}, 1 \leq i \leq k$, we get since $u \leq 1$ and $M(s, h)=M(s, u)$ that $M\left(r_{k}, u\right) \leq \beta^{k}$. Since $\bar{M}$ is nondecreasing it follows for $r_{k+1} \leq r \leq r_{k}$ that

$$
M(r, u) \leq M\left(r_{k}, u\right) \leq c(r / R)^{\sigma}
$$

where $\beta=\delta^{\sigma}$. From (2.11) with $s=8 r, s_{1}=\frac{1}{2} s$, and the fact that

$$
K_{1}\left(x_{1}\right) \leq q\left(x_{1}\right)=h\left(x_{1}\right) \leq M(8 r, u)
$$

we deduce

$$
\left[r^{(\alpha p-n)} \mu\left(B\left(x_{1}, r\right)\right)\right]^{1 /(p-1)} \leq c M(8 r, u), \quad 0<r<\infty .
$$

Hence,

$$
\left[r^{(\alpha p-n)} \mu\left(B\left(x_{1}, r\right)\right)\right]^{1 /(p-1)} \leq c(r / R)^{\sigma}, \quad 0<r<R .
$$

We note that $x_{1} \in F$ is arbitrary in (2.14) subject to the requirement that $P\left(x_{1}\right)=$ 1 , which from $(\mathrm{C})$ of $\S 1$ is true $(\alpha, p)$ q.e. on $\operatorname{supp} \mu$. We claim that $(2.14)$ holds whenever $x_{1} \in \mathbf{R}^{n}$ for some positive constant $c$. To see this we note first from a 
boundedness principle (see $[\mathbf{2}, \mathbf{9}]$ ) that $P$ is bounded above by a positive constant in $\mathbf{R}^{n}$ depending only on $\alpha, p$, and $n$. From the definition of $\operatorname{supp} \mu$ and (B) of $\S 1$ it follows that for $r>0$

$$
R_{\alpha, p}[B(x, r) \cap \operatorname{supp} \mu] \neq 0, \quad x \in \operatorname{supp} \mu \text {. }
$$

From this inequality we easily deduce that (2.14) holds for all $x_{1} \in \operatorname{supp} \mu$. An easy argument using the compactness of supp $\mu$ now shows that (2.14) holds for all $x_{1} \in \mathbf{R}^{n}$. We also note from uniform fatness of $F$ and (C) of $\S 1$ that

$$
\mu[F] \geq c \lambda R^{(n-\alpha p)} .
$$

Let $0<\varepsilon<\sigma(p-1)$ and let $\beta>0, q>1$, be fixed numbers with $\alpha p-\varepsilon \leq \beta q<\alpha p$. Put

$$
\nu(G)=R^{(\alpha p-\beta q)} \mu(G),
$$

when $G$ is a Borel set. Then (2.14) implies (see [1, Theorem 2]) that $I_{\beta} *$ $\left(I_{\beta} * \nu\right)^{1 /(q-1)} \leq c \gamma$, where $\gamma$ depends only on $\alpha, p, n$, and $\lambda$. This inequality, (B) of $\S 1,(2.15)$, and the fact that $F \subseteq E \cap \bar{B}\left(x_{0}, R\right)$ imply (1.3) of Theorem 1. To prove (1.4) let $B\left(x_{j}, r_{j}\right), j=1, \ldots$, be a covering of $F$ by balls with $r_{j} \leq R$, $j=1,2 \ldots$ Then from $(2.14)$ and $(2.15)$ we get

$$
\begin{gathered}
\sum r_{j}^{(n-\alpha p+\sigma(p-1))} \geq c R^{\sigma(p-1)} \sum \mu\left(B\left(x_{j}, r_{j}\right)\right) \\
\geq c R^{\sigma(p-1)} \mu(F) \geq c R^{(n-\alpha p)+\sigma(p-1)} .
\end{gathered}
$$

Taking the infimum of the left-hand sum over all such coverings we get (1.4) of Theorem 1 with $\varepsilon=\sigma(p-1)$. This completes the proof of Theorem 1 for $\alpha \geq 2$.

3. Proof of Theorem 1 for $0<\alpha<2$. We now suppose that $0<\alpha p<n$, $0<\alpha<2$, and shall use the same notation as in $\S 2$. The proof of Theorem 1 in this case is harder since now the equilibrium potential $P$ for $F$ need not be superharmonic in the classical sense. However $P$ is still $\alpha$ superharmonic in the sense of Landkof [12], and $u=1-P$ may be written in the form $u=h-q$ in $B\left(x_{1}, s\right)$ where $q$ is an $\alpha$ Green's potential and $h \geq u$ is the least $\alpha$ harmonic majorant of $u$ in $B\left(x_{1}, s\right)$. Moreover

$$
h(x)=\int_{\mathbf{R}^{n}-B\left(x_{1}, s\right)} Q_{s}(x, y) u(y) d y
$$

(see [12, Chapter 1, §6, p. 123]) where

$$
Q_{s}(x, y)=\frac{c\left(s^{2}-\left|x_{1}-x\right|^{2}\right)^{\alpha / 2}}{\left(\left|y-x_{1}\right|^{2}-s^{2}\right)^{\alpha / 2}}|x-y|^{-n}, \quad x \in B\left(x_{1}, s\right), y \in \mathbf{R}^{n}-B\left(x_{1}, s\right),
$$

and $c$ is chosen so that the integral in (3.1) is one when $u \equiv 1$. For fixed $s \leq R$ and $0<s_{1}<\frac{1}{2} s$, we first show, as in $\S 2$, that if

$$
\left|h(x)-h\left(x_{1}\right)\right| \leq \frac{1}{4} M(s, h), \quad x \in B\left(x_{1}, s_{1}\right),
$$

then there exists $\beta, \frac{3}{4} \leq \beta<1$, depending only on $\alpha, p$, and $\lambda$ such that

$$
M\left(\frac{1}{8} s_{1}, u\right) \leq \beta M(s, h)
$$


Recall that $M(s, h)=\sup _{x \in B\left(x_{1}, s\right)} h(x)$. To prove (3.3) we follow closely the proof of (2.4). Observe from (3.2) that if $h\left(x_{1}\right) \leq \frac{1}{2} M(s, h)$, then (3.3) holds with $\beta=\frac{3}{4}$ since $u \leq h$ in $B\left(x_{1}, s\right)$. Otherwise, from (C) of $\S 1$ and (3.2) we deduce

$$
q(x) \geq h(x) \geq \frac{1}{4} M(s, h), \quad(\alpha, p) \text { q.e. },
$$

on $F \cap B\left(x_{1}, s_{1}\right)$. Now

$$
\begin{aligned}
q(x) & =c \int_{B\left(x_{1}, s\right)} g(x, t)\left(I_{\alpha} * \mu\right)^{1 /(p-1)}(t) d t \\
& =c \int_{B\left(x_{1}, s_{1} / 2\right)} \cdots+c \int_{B\left(x_{1}, s\right)-B\left(x_{1}, s_{1} / 2\right)} \cdots \\
& =K_{1}+K_{2} .
\end{aligned}
$$

Here $g(x, \cdot)$ is the $\alpha$ Green's function for $B\left(x_{1}, s\right) . g$ can be written explicitly as (see [12, Chapter 4, §5])

$$
g(x, t)=|x-t|^{(\alpha-n)}-\int_{\mathbf{R}^{n}-B\left(x_{1}, s\right)} Q_{s}(x, y)|t-y|^{(\alpha-n)} d y
$$

when $t \in B\left(x_{1}, s\right)$ and $x \in \mathbf{R}^{n}$. We note that $g(\cdot, t) \equiv 0$ in $\mathbf{R}^{n}-\bar{B}\left(x_{1}, s\right), g \geq 0$, and $g$ is $\alpha$ harmonic as a function of $x$ in $B\left(x_{1}, s_{1} / 2\right)$ when $t \in B\left(x_{1}, s\right)-B\left(x_{1}, s_{1} / 2\right)$. Equivalently, (3.1) holds in $B\left(x_{1}, s_{1} / 2\right)$ with $u, h$ replaced by $g(\cdot, t)$ and $s$ by $\frac{1}{2} s_{1}$. Hence $K_{2}$ is a positive $\alpha$ harmonic function in $B\left(x_{1}, \frac{1}{2} s_{1}\right)$ and so satisfies a Harnacktype inequality in $B\left(x_{1}, \frac{1}{4} s_{1}\right)$, as we see from (3.1). Thus either $K_{2} \leq \frac{1}{8} M(s, h)$ or $K_{2} \geq c M(s, h)$ in $B\left(x_{1}, \frac{1}{4} s_{1}\right)$. In the second case we see that (3.3) holds. In the first case we find from (3.4) that

$$
K_{1} \geq \frac{1}{8} M(s, h), \quad(\alpha, p) \text { q.e. }
$$

on $B\left(x_{1}, \frac{1}{4} s_{1}\right) \cap F$.

We claim for $x \in B\left(x_{1}, s_{1} / 4\right)$ and $t \in B\left(x_{1}, s_{1} / 2\right)$ that

$$
c|x-t|^{(\alpha-n)} \leq|x-t|^{(\alpha-n)}-(s / 2)^{(\alpha-n)} \leq g(x, t) .
$$

The left inequality is easily verified while the right inequality is true because the difference of the above functions is $\alpha$ harmonic in $B\left(x_{1}, s / 2\right)$ and $\leq 0$ on $\mathbf{R}^{n}-$ $B\left(x_{1}, s / 2\right)$. Moreover, clearly

$$
g(x, t) \leq|x-t|^{(\alpha-n)}, \quad x, t \in B\left(x_{1}, s\right) .
$$

Using (3.6), (3.7) in place of (2.6), (2.7), we repeat the argument leading to the proof of (2.4). For completeness we give some details. Let $\mu_{1}=\mu / B\left(x_{1}, \frac{1}{4} s_{1}\right)$ and $\mu_{2}=\mu-\mu_{1}$. Put

$$
L_{i}(x)=2^{1 /(p-1)} \int_{B\left(x_{1}, s_{1} / 2\right)}|x-t|^{(\alpha-n)}\left(I_{\alpha} * \mu_{i}\right)^{1 /(p-1)}(t) d t, \quad i=1,2 .
$$

Then from (3.6)-(3.7) we see that $K_{1} \leq L_{1}+L_{2}$ in $B\left(x_{1}, s_{1} / 2\right)$ and $K_{1} \geq c L_{2}$ in $B\left(x_{1}, s_{1} / 4\right)$. As in $\S 2, L_{2}$ satisfies a Harnack inequality in $B\left(x_{1}, s_{1} / 8\right)$ so as above either (3.3) holds or $L_{2} \leq \frac{1}{16} M\left(s_{1}, h\right)$ in $B\left(x_{1}, s_{1} / 8\right)$. If the last inequality holds, then from (3.5) we deduce

$$
\frac{1}{16} M(s, h) \leq L_{1} \leq c I_{\alpha} *\left(I_{\alpha} * \mu_{1}\right)^{1 /(p-1)},
$$


$(\alpha, p)$ q.e., on $F \cap B\left(x_{1}, \frac{1}{8} s_{1}\right)$. It follows that (compare with (2.10))

$$
R_{\alpha, p}\left[F \cap B\left(x_{1}, \frac{1}{8} s_{1}\right)\right] M(s, h)^{p} \leq c \int_{\mathbf{R}^{n}} I_{\alpha} *\left(I_{\alpha} * \mu_{1}\right)^{1 /(p-1)} d \mu_{1} .
$$

Now

$$
\left[\mu_{1}\left(\mathbf{R}^{n}\right) s_{1}^{(\alpha p-n)}\right]^{1 /(p-1)} \leq c I_{\alpha} *\left(I_{\alpha} * \mu_{1}\right)^{1 /(p-1)} \leq c K_{1}
$$

in $B\left(x_{1}, s_{1} / 4\right)$ as follows easily from (3.6). Also

$$
K_{1} \leq q=h,
$$

$(\alpha, p)$ q.e. in $\operatorname{supp} \mu_{1}$.

Using (3.9), (3.10) in (3.8) it follows that

$$
\left\{s_{1}^{(\alpha p-n)} R_{\alpha, p}\left[F \cap B\left(x_{1}, s_{1} / 8\right)\right]\right\}^{1 /(p-1)} M(s, h) \leq c K_{1} \leq c q
$$

in $B\left(x_{1}, s_{1} / 4\right)$. Using uniform fatness of $F$ we get (3.3), as in $\S 2$.

(3.3) implies as in $\S 2$ that there exists $\delta, 0<\delta<\frac{1}{32}$, such that if $r_{j}=\delta^{j} R$, $j=0,1, \ldots$, then

$$
M\left(r_{j}, u\right) \leq \beta^{j}
$$

The proof is more difficult in this case though since $h$ cannot be estimated above in $B\left(x_{1}, r\right)$ by $M(r, u)$. Observe that (3.11) is trivially true when $j=0$. To prove (3.11) we first allow $\delta$ to vary and shall later fix it at a number satisfying several conditions. To see the requirements on $\delta$ suppose for fixed $k \geq-1$ that we have chosen $r_{0}, \ldots, r_{k+1}$, such that (3.11) holds. Let $s=r_{k+1}, 0<\delta^{\alpha}<\frac{1}{2}$, and put $u^{+}=\max (u, 0)$ as previously. Define $h$ relative to $u$ as in (3.1). Then from (3.1), the fact that $0=u\left(x_{1}\right) \leq h\left(x_{1}\right)$, and (3.11), we obtain for $k \geq 1$ and $x \in B\left(x_{1}, s / 2\right)$

$$
\begin{aligned}
|\nabla h(x)| & \leq \frac{c}{s} \int_{\mathbf{R}^{n}-B\left(x_{1}, s\right)} Q_{s}\left(x_{1}, y\right)|u(y)| d y \\
& \leq \frac{2 c}{s} \int_{\mathbf{R}^{n}-B\left(x_{1}, s\right)} Q_{s}\left(x_{1}, y\right) u^{+}(y) d y \\
& \leq \frac{c}{s}\left[\beta^{k}+\sum_{j=0}^{k-1} \beta^{j} \int_{\mathbf{R}^{n}-B\left(x_{1}, r_{j+1}\right)} Q_{s}\left(x_{1}, y\right) d y\right] \\
& \leq \frac{c}{s}\left[\beta^{k}+s^{\alpha} \sum_{j=0}^{k-1} \beta^{j} \int_{\mathbf{R}^{n}-B\left(x_{1}, r_{j+1}\right)}\left|y-x_{1}\right|^{-(n+\alpha)} d y\right] \\
& \leq \frac{c}{s}\left[\beta^{k}+\sum_{j=0}^{k-1} \beta^{j}\left(\frac{s}{r_{j+1}}\right)^{\alpha}\right] \leq \frac{c}{s}\left[\beta^{k}+\sum_{j=0}^{k-1} \beta^{j}\left(\delta^{\alpha}\right)^{(k-j)}\right] \\
& \leq \frac{c}{s}\left[\beta^{k}+\beta^{k-1} \delta^{\alpha}\right] \leq \frac{c_{1} \beta^{k}}{s},
\end{aligned}
$$

where we have used the fact that $\delta^{\alpha} \leq \frac{1}{2}<\frac{3}{4} \leq \beta$. If $k=-1,0$, the above inequality also holds for $c_{1}$ large enough as is easily seen. We now fix $\delta$ to be the smaller of the numbers: $\frac{1}{32},\left(\frac{1}{2}\right)^{1 / \alpha}$, and $1 / 250 c_{1}$, where $c_{1}$ is the constant in (3.12). 
Using (3.12), the fact that $\frac{3}{4} \leq \beta<1$, and the mean value theorem we find for $s_{1}=8 \delta s$ that

$$
\left|h(x)-h\left(x_{1}\right)\right| \leq \frac{1}{4} \beta^{k+2}, \quad x \in B\left(x_{1}, s_{1}\right) .
$$

If $M(s, h) \leq \beta^{k+2}$, then (3.11) holds for $j=k+2$ since $s=r_{k+1}>r_{k+2}$ and $u \leq h$ in $B\left(x_{1}, s\right)$. Otherwise from (3.13) we see that (3.2) holds. Using (3.3) and the induction hypothesis we again see that (3.11) holds for $j=k+2$. We conclude by induction that (3.11) is valid.

From (3.11) we find as in $\S 2$ that there exists $\sigma_{1}>0$ with

$$
M(r, u) \leq c(r / R)^{\sigma_{1}}, \quad 0<r<R .
$$

If $\sigma=\min \left(\sigma_{1}, \alpha\right)$, it follows from the above inequality and (3.1) as in the proof of (3.12) that $h\left(x_{1}\right) \leq c(s / R)^{\sigma}$. From this last inequality, (3.9), and (3.10) we see that (2.14) holds also when $0<\alpha<2$. Theorem 1 follows from (2.14) just as in $\S 2$. We omit the details.

4. Proof of Theorem 1 for $\alpha p=n$. Let $J_{\alpha}$ be the truncated Riesz kernel defined in $\S 1$ and for $\alpha p=n$ let $P=J_{\alpha} *\left(J_{\alpha} * \mu\right)^{1 /(p-1)}$ be the equilibrium potential for

$$
F_{1}=\left\{x_{0}+R^{-1}\left(y-x_{0}\right): y \in F\right\} \text {. }
$$

Let

$$
P_{1}(x)=\int_{B\left(x_{0}, 50\right)} J_{\alpha}(x-y)\left(J_{\alpha} * \mu\right)^{1 /(p-1)}(y) d y, \quad x \in \mathbf{R}^{n}
$$

From the definition of $P$ and $P_{1}$ we see that

$$
\left|\nabla\left(P-P_{1}\right)\right|(x) \leq c, \quad x \in B\left(x_{0}, 2\right) .
$$

Let $u=1-P$ and for fixed $x_{1} \in \operatorname{supp} \mu \subseteq \bar{B}\left(x_{0}, 1\right)$ with $P\left(x_{1}\right)=1$ put $u_{1}=$ $P_{1}\left(x_{1}\right)-P_{1}$. From the above inequality we see that

$$
\left|u_{1}-u\right|(x) \leq\left|\left(P_{1}-P\right)(x)-\left(P_{1}-P\right)\left(x_{1}\right)\right| \leq c_{2}\left|x-x_{1}\right|,
$$

when $x \in B\left(x_{0}, 2\right)$.

We note that $u_{1}$ is subharmonic in $B\left(x_{0}, 2\right)$ when $\alpha \geq 2$ and $u_{1}$ is $\alpha$ subharmonic in $B\left(x_{0}, 2\right)$ when $0<\alpha<2$. For fixed $s, 0<s \leq 1$, we write $u_{1}=h_{1}-q_{1}$, where $h_{1}$ is the least harmonic majorant of $u_{1}$ in $B\left(x_{1}, s\right)$ when $\alpha \geq 2$ and $h_{1}$ is the least $\alpha$ harmonic majorant of $u_{1}$ in $B\left(x_{1}, s\right)$, defined as in (3.1), when $0<\alpha<2$. Also, $q_{1}$ is a Green's potential or $\alpha$ Green's potential in $B\left(x_{1}, s\right)$ depending on whether $\alpha \geq 2$ or $0<\alpha<2$. If $0<s_{1} \leq s / 2$,

$$
M\left(s, h_{1}\right) \geq 8 c_{2} s,
$$

where $c_{2}>1$ is the constant in (4.1), and

$$
\left|h_{1}(x)-h_{1}\left(x_{1}\right)\right| \leq \frac{1}{4} M(s, h)
$$

in $B\left(x_{1}, s_{1}\right)$.

We show as in $\S \S 2-3$ that there exists $\beta, \frac{3}{4} \leq \beta<1$, such that

$$
M\left(s_{1} / 8, u_{1}\right) \leq \beta M\left(s, h_{1}\right) .
$$


As before we note from (4.3) that either $h_{1}\left(x_{1}\right) \leq \frac{1}{2} M\left(s, h_{1}\right)$ in which case (4.4) holds with $\beta=\frac{3}{4}$ or $h_{1} \geq \frac{1}{4} M\left(s, h_{1}\right)$ in $B\left(x_{1}, s_{1}\right)$. In this case from (4.2), (4.1) and (C) of $\S 1$ we find for $x \in F_{1} \cap B\left(x_{1}, s_{1}\right)$

$$
0 \geq u(x) \geq u_{1}(x)-c_{2} s \geq \frac{1}{8} M\left(s, h_{1}\right)-q_{1}(x) .
$$

Hence

$$
q_{1} \geq \frac{1}{8} M(s, h), \quad(\alpha, p) \text { q.e., on } F_{1} \cap B\left(x_{1}, s_{1}\right) .
$$

We define $K_{i}, L_{i}, i=1,2$, as in $\S \S 2$ and 3 with $I_{\alpha}$ replaced by $J_{\alpha}$ and $u$ by $u_{1}$. Proceeding as in $\S \S 2$ and 3 we find that either (4.4) holds or

$$
L_{1} \geq \frac{1}{32} M\left(s, h_{1}\right), \quad(\alpha, p) \text { q.e on } F_{1} \cap B\left(x_{1}, \frac{1}{8} s_{1}\right) .
$$

Let $H(x)=L_{1}\left[x_{1}+s_{1}\left(x-x_{1}\right)\right], x \in B\left(x_{1}, 1\right)$, and put

$$
\nu(T)=\mu_{1}\left(s_{1} T\right), \quad T \text { a Borel set, }
$$

where $s_{1} T=\left\{x_{1}+s_{1}\left(y-x_{1}\right): y \in T\right\}$ and $\mu_{1}=\mu \mid B\left(x_{1}, s_{1} / 4\right)$.

Changing variables in the integrals defining $L_{1}$ in $\S \S 2$ and 3 we see that

$$
H(x) \leq c\left[J_{\alpha} *\left(J_{\alpha} * \nu\right)^{1 /(p-1)}\right](x), \quad x \in B\left(x_{1}, 1\right) .
$$

Using (4.6), (4.7), and (1.1) we deduce as in $\S \S 2$ and 3 (see (3.8) and (2.10)) that

$$
\lambda M\left(s, h_{1}\right)^{p} \leq c \int_{\mathbf{R}^{n}} J_{\alpha} *\left(J_{\alpha} * \nu\right)^{1 /(p-1)} d \nu .
$$

Again as in $\S \S 2$ and 3 we find

$$
J_{\alpha} *\left(J_{\alpha} * \nu\right)^{1 /(p-1)}(x) \leq c L_{1}\left[x_{1}+s_{1}\left(x-x_{1}\right)\right], \quad x \in B\left(x_{1}, \frac{1}{4}\right) .
$$

Also from (4.1) and (C) of $\S 1$, we have

$$
L_{1} \leq c K_{1} \leq c q_{1} \leq c h_{1}+c c_{2} s,
$$

$(\alpha, p)$ q.e. on supp $\mu_{1}$. Using these inequalities and (4.2) we deduce as previously first that

$$
\lambda^{1 /(p-1)} M\left(s, h_{1}\right) \leq c q_{1}, \quad \text { in } B\left(x_{1}, \frac{1}{4}\right)
$$

and thereupon that (4.4) holds.

(4.4) implies there exists $\delta, 0<\delta<\frac{1}{32}$, such that if $r_{j}=\delta^{j}, j=0,1, \ldots$, and $c_{2}>1$ is the constant in (4.1), then

$$
M\left(r_{j}, u_{1}\right) \leq 2 c_{2} \beta^{j}, \quad j=0,1, \ldots
$$

In fact if $(4.8)$ holds for $r_{0}, r_{1}, \ldots, r_{k+1}(k \geq-1)$ and $s=r_{k+2}$, then either

$$
M\left(s, h_{1}\right) \leq 2 c_{2} \beta^{k+2}
$$

(in which case (4.8) holds with $j=k+2$ since $u_{1} \leq h_{1}$ ) or (4.2) is satisfied since $4 \delta<\beta$. If (4.2) is true, we argue as in $\S \S 2$ and 3 with $u$ replaced by $u_{1}$ to get (4.8) for $j=k+2$. Then by induction we obtain (4.8). The rest of the proof of Theorem 1 for $\alpha p=n$ is the same as in the previous sections. The proof of Theorem 1 is now complete. 
5. Proof of Theorem 2. Let $D$ be $(1, p)$ uniformly fat, $1<p \leq n$, and let $\left\{Q_{i}\right\}_{1}^{\infty}$ be a sequence of Whitney cubes for $D$ with $Q_{i} \subseteq D, i=1,2, \ldots$, and

$$
(4 n)^{-1} d\left(Q_{i}, \partial D\right) \leq r_{i} \leq d\left(Q_{i}, \partial D\right),
$$

where $r_{i}, i=1,2, \ldots$, denotes the sidelength of $Q_{i}$ and $d(E, F)$ denotes the distance from the set $E$ to the set $F$. Let $\tilde{Q}_{i}$ denote the cube with the same center as $Q_{i}$ and with sidelength, $100 n r_{i}$. Given $u \in C_{0}^{\infty}(D)$ put $v=|u|$. Let $h_{Q}$ denote the average of the function $h$ over the cube $Q$. We note that (see [15, p. 195])

$$
\left|v(x)-v_{Q}\right| \leq c \int_{Q}|\nabla v|(y)|x-y|^{(1-n)} d y, \quad x \in Q .
$$

From (5.2) it can be shown that

$$
\int_{Q}\left|v(x)-v_{Q}\right|^{p} d x \leq c r^{p} \int_{Q}|\nabla v|^{p} d x
$$

where $r$ is the sidelength of $Q$.

Observe from (5.1) that $d(x, \partial D) \geq c r_{i}$, when $x \in Q_{i}$. Using this observation and (5.3) with $Q=Q_{i}, i=1,2, \ldots$, and $d(x)=d(\{x\}, \partial D)$, we obtain

$$
\begin{aligned}
\int_{D}\left|\frac{u}{d}\right|^{p} d x & \leq c \sum\left(r_{i}^{-p} \int_{Q_{i}} v^{p} d x\right) \\
& \leq c\left[\sum\left(r_{i}^{(n-p)} v_{Q_{i}}^{p}\right)+\int_{D}|\nabla v|^{p} d x\right] .
\end{aligned}
$$

To estimate $v_{Q_{i}}$ choose $x_{i} \in \partial D$ such that $d\left(\left\{x_{i}\right\}, Q_{i}\right)=d\left(Q_{i}, \partial D\right)$. Note from (5.1) and the definition of $\tilde{Q}_{i}$ that

$$
\bar{B}\left(x_{i}, r_{i}\right) \subseteq \tilde{Q}_{i}, \quad i=1,2, \ldots
$$

Let $q=p-\varepsilon / 2$, where $\varepsilon$ is as in Theorem 1 for $\alpha=1$, and put $q^{\prime}=q /(q-1)$. Let $\mu$ be a $R_{1, q}$ capacitary measure for $\bar{B}\left(x_{i}, r_{i}\right) \cap\left(\mathbf{R}^{n}-D\right)$. Then from (5.2) with $Q=\tilde{Q}_{i}$, the fact that $v=0$ on $\mathbf{R}^{n}-D$, and (C) of $\S 1$, we obtain

$$
\begin{aligned}
& v_{\tilde{Q}_{i}} \mu\left[\bar{B}\left(x_{i}, r_{i}\right)\right.\left.\cap\left(\mathbf{R}^{n}-D\right)\right] \\
& \leq c \int_{\tilde{Q}_{i}}|\nabla v|\left(I_{1} * \mu\right) d y \leq c\left\||\nabla v| \chi_{i}\right\|_{q}\left\|I_{1} * \mu\right\|_{q^{\prime}} \\
& \leq c\left\||\nabla v| \chi_{i}\right\|_{q}\left\{\mu\left[\bar{B}\left(x_{i}, r_{i}\right) \cap\left(\mathbf{R}^{n}-D\right)\right]\right\}^{1 / q^{\prime}}
\end{aligned}
$$

where $\chi_{i}$ denotes the characteristic function of $\tilde{Q}_{i}, i=1,2, \ldots$ Observe from Theorem 1 that

$$
\mu\left[\bar{B}\left(x_{i}, r_{i}\right) \cap\left(\mathbf{R}^{n}-D\right)\right] \geq c r_{i}^{n-q} .
$$

Using this inequality in (5.5) and doing some arithmetic we find that

$$
v_{\tilde{Q}_{i}}^{p} r_{i}^{(n-p)} \leq c\left\||\nabla v| \chi_{i} r_{i}^{-n / q}\right\|_{q}^{p} r_{i}^{n} \leq c\left(f_{\tilde{Q}_{i}}\right)^{p / q} r_{i}^{n}
$$

where $f=|\nabla v|^{q}$. Using (5.6) and $v_{Q_{i}} \leq c v_{\tilde{Q}_{i}}$ in (5.4) we conclude that

$$
\int_{D}\left|\frac{u}{d}\right|^{p} d x \leq c\left[\sum\left(f_{\tilde{Q}_{i}}\right)^{p / q} r_{i}^{n}+\int_{D}|\nabla v|^{p} d x\right] .
$$


We observe that $\left\{\tilde{Q}_{i}\right\}$ is a sequence of "Carleson" cubes. That is, if $Q$ is a cube

$$
\sum_{\tilde{Q}_{i} \subseteq Q} H^{n}\left(\tilde{Q}_{i}\right) \leq c \sum_{Q_{i} \subseteq Q} r_{i}^{n} \leq c H^{n}(Q) .
$$

Also if $\tau=p / q>1$, then $f \in L^{\tau}\left(\mathbf{R}^{n}\right)$. Applying a well-known lemma originally proved by Carleson when $p=2, n=1$ (see [7, 11, and 19, Chapter 7, ex. 4.4]), we obtain

$$
\sum\left(f_{\tilde{Q}_{i}}\right)^{p / q} r_{i}^{n} \leq c\|f\|_{\tau}^{\tau}=c \int_{D}|\nabla v|^{p} d x .
$$

Using this inequality in (5.7) and the fact that $|\nabla u|=|\nabla v|$ almost everywhere, we obtain Theorem 2.

As mentioned in $\S 1,(1.5)$ holds for any open set $D \neq \mathbf{R}^{n}$ when $p>n$. In fact if $u \in C_{0}^{\infty}(D)$ and $q=n+\frac{1}{2}(p-n)$, then from (5.2) with $u=v$ and Hölder's inequality we obtain

$$
|u(x)-u(y)| \leq c\||\nabla u| \chi\|_{q} r^{(1-n / q)}, \quad x, y \in Q,
$$

where again $Q$ is a cube with sidelength $r$ and $\chi$ denotes the characteristic function of $Q$.

Defining $\left(\tilde{Q}_{i}\right)$ as before and applying the above inequality with $Q=\tilde{Q}_{i}$ and $u(y)=0$ we see that (5.6) is still true. Repeating the argument in Theorem 3 from (5.6) on we conclude that (1.5) holds whenever $D \neq \mathbf{R}^{n}$.

6. Proof of Theorem 3. We first show that Theorem 2 has a converse when $p=n$. The proof is by contradiction. Suppose that $D$ is an open set and $\mathbf{R}^{n}-D$ is not $(1, n)$ uniformly fat. Then for each $\varepsilon>0$ there exists $x_{0}=x_{0}(\varepsilon)$ in $\mathbf{R}^{n}-D$ and $r=r(\varepsilon)>0$ such that if

$$
\Lambda=\left\{x_{0}+r^{-1}\left(y-x_{0}\right): y \in \bar{B}\left(x_{0}, r\right) \cap\left(\mathbf{R}^{n}-D\right)\right\},
$$

then

$$
R_{1, n}(\Lambda) \leq \varepsilon^{n}
$$

Let

$$
\Gamma(\Lambda)=\inf _{\phi}\left(\int|\nabla \phi|^{n} d x\right)
$$

where the infimum is taken over all $\phi \in C_{0}^{\infty}\left(B\left(x_{0}, 4\right)\right)$ with $\phi \equiv 1$ in a neighborhood of $\Lambda$. It is well known (see [4]) that

$$
c^{-1} \Gamma(\Lambda) \leq R_{1, n}(\Lambda) \leq c \Gamma(\Lambda)
$$

Using (6.1), (6.2), we see there exists $\psi \in C_{0}^{\infty}\left(B\left(x_{0}, 4\right)\right)$ with $\psi \equiv 1$ in an open set containing $\Lambda$ and

$$
\int_{\mathbf{R}^{n}}|\nabla \psi|^{n} d x \leq c \varepsilon^{n} .
$$

Let $\theta \in C_{0}^{\infty}\left[B\left(x_{0}, 1\right)\right]$ with $\theta \equiv 1$ on $B\left(x_{0}, \frac{1}{2}\right)$ and $|\nabla \theta| \leq 1000$. Put

$$
h(x)=(1-\psi(x)) \theta(x), \quad x \in \mathbf{R}^{n},
$$


and observe that $u(x)=h\left(x_{0}+\left(x-x_{0}\right) / r\right), x \in \mathbf{R}^{n}$, is in $C_{0}^{\infty}(D)$. Also,

$$
\begin{aligned}
\int_{\mathbf{R}^{n}}|\nabla u|^{n} d x & =\int_{\mathbf{R}^{n}}|\nabla h|^{n} d x \\
& \leq c\left[\int_{B\left(x_{0}, 1\right)}(1-\psi)^{n} d x+\int_{B\left(x_{0}, 1\right)}|\nabla \psi|^{n} d x\right] \leq c,
\end{aligned}
$$

where we have used Poincaré's inequality, and (6.3). Again from these inequalities we deduce

$$
\begin{aligned}
H^{n}\left[\left\{x: \psi(x) \geq \frac{1}{2}\right\}\right] & \leq 2^{n} \int \psi^{n} d x \\
& \leq c \int_{B\left(x_{0}, 4\right)}|\nabla \psi|^{n} d x \leq c \varepsilon^{n} .
\end{aligned}
$$

Let $G \subseteq \mathbf{R}$ be the set of all $t \in\left(0, \frac{1}{2}\right)$ for which

$$
H^{n-1}\left[\left\{x: \psi(x)<\frac{1}{2}\right\} \cap \partial B\left(x_{0}, t\right)\right] \geq \frac{1}{2} H^{n-1}\left[\partial B\left(x_{0}, t\right)\right] .
$$

From Fubini's Theorem and (6.5) we find first that there exists $c_{3}>0$ with

$$
\int_{(0,1 / 2)-G} t^{n-1} d t \leq\left(c_{3} \varepsilon\right)^{n}
$$

and thereupon since $t \rightarrow t^{-n}$ is decreasing that

$$
\int_{G} t^{-1} d t=\int_{G} t^{-n} t^{n-1} d t \geq \int_{c_{3} \varepsilon}^{1 / 2} t^{-1} d t=-\ln \left(2 c_{3} \varepsilon\right) .
$$

Now if $d(x)=d\left(\{x\}, \mathbf{R}^{n}-D\right)$, then from (6.6) and the fact that $h \geq \frac{1}{2}$ on $G$ we obtain

$$
\begin{aligned}
\int_{D}\left(\frac{u}{d}\right)^{n} d x & \geq \int_{B\left(x_{0}, r\right)} \frac{u(x)^{n}}{\left|x-x_{0}\right|^{n}} d x=\int_{B\left(x_{0}, 1\right)} \frac{h(x)^{n}}{\left|x-x_{0}\right|^{n}} d x \\
& \geq c \int_{G} t^{-1} d t \geq-c \ln \left(2 c_{3} \varepsilon\right) .
\end{aligned}
$$

Given $A>0$ we see from (6.4) and (6.7) that for $\varepsilon>0$, sufficiently small, there exists $u \in C_{0}^{\infty}(D)$ for which (1.5) is false. This proves Theorem 3 for $p=n$.

To prove Theorem 3 for $1<p<n$ requires an explicit construction. To this end let $\left(m_{j}\right)_{1}^{\infty}$ be an increasing sequence of positive integers with $m_{j} \geq j+1, j=1, \ldots$, to be chosen later. Put $s_{0}=1 / 2 n$ and for $j \geq 1$, let

$$
s_{j}=s_{j-1}\left(m_{j}\right)^{-j}, \quad t_{j}=\left(s_{j-1}-s_{j}\right) /\left(m_{j}-1\right) .
$$

Since $m_{j} \geq j+1$ it is easily seen that

$$
t_{j}-10 s_{j} \geq\left(2 m_{j}\right)^{-1} s_{j-1}, \quad j=10,11, \ldots
$$

If $J=[a, b]$ is an interval with $b-a=s_{j-1}$, let $\Phi\left(J, m_{j}\right)$ be the $m_{j}$ equally spaced closed intervals of length $s_{j}$ contained in $J$ defined by

$$
\Phi\left(J, m_{j}\right)=\left\{\left[a+(i-1) t_{j}, a+(i-1) t_{j}+s_{j}\right]: 1 \leq i \leq m_{j}\right\} .
$$

Define families $K_{j}$ of closed intervals inductively as follows:

$$
K_{0}=[-1 / 4 n, 1 / 4 n], \quad K_{j}=\left\{\Phi\left(J, m_{j}\right): J \in K_{j-1}\right\}, \quad j \geq 1 .
$$


Let $L_{j}, j=0,1, \ldots$, be the family of cubes defined by

$$
L_{j}=\left\{Q=J_{1} \times \cdots \times J_{n}: J_{i} \in K_{j}, 1 \leq i \leq n\right\} .
$$

Note that $L_{j}, j=i, \ldots$, consists of $\left(m_{1} \cdots m_{j}\right)^{n}$ closed cubes of sidelength $s_{j}$. Also if $Q \in L_{j-1}$, then

$$
\sum_{\substack{T \in L_{j} \\ T \subseteq Q}}\left[H^{n}(T)\right]^{1 / j}=H^{n}(Q)^{1 / j}, \quad j=1,2, \ldots
$$

Let

$$
F_{j}=\bigcup_{Q \in L_{j}} Q, \quad j=0,1, \ldots, \quad F=\bigcap_{0}^{\infty} F_{j} .
$$

Let $D_{j}=B(0,1)-F_{j}, j=0,1, \ldots$, and $D=B(0,1)-F$. If $x \in D_{j}$ let

$$
\tilde{u}_{j}(x)=\left\{\begin{array}{l}
0 \quad \text { when } d\left(\{x\}, \partial D_{j}\right)<s_{j}, \\
s_{j}^{-1}\left[d\left(\{x\}, \partial D_{j}\right)-s_{j}\right], \quad \text { when } s_{j} \leq d\left(\{x\}, \partial D_{j}\right) \leq 2 s_{j}, \\
1 \quad \text { when } d\left(\{x\}, \partial D_{j}\right)>2 s_{j} .
\end{array}\right.
$$

Put $u_{j}=\left[\tilde{u}_{j} * \theta_{j}\right] \theta, j=0,1, \ldots$, where

$$
\theta_{j}(x)=\left(2 / s_{j}\right)^{n} \theta\left[2 x / s_{j}\right]\left(\|\theta\|_{1}\right)^{-1}, \quad x \in \mathbf{R}^{n},
$$

and as previously, $\theta \in C_{0}^{\infty}(B(0,1))$ with $\theta \equiv 1$ on $B\left(0, \frac{1}{2}\right)$. From the definition of $\tilde{u}_{j}$ we see that $u_{j} \in C_{0}^{\infty}\left(D_{j}\right)$,

$$
\nabla u_{j}(x)=0 \text { if } d\left(\{x\}, \partial D_{j}\right) \geq 3 s_{j} \text { and }|x|<\frac{1}{2},
$$

while $\nabla u_{j}=\nabla \tilde{u}_{j} * \theta_{j}, j=0,1, \ldots$ Using these facts we find that

$$
\int_{D_{j}}\left|\nabla u_{j}\right|^{p} d x \leq c\left(m_{1} \cdots m_{j}\right)^{n}\left(s_{j}\right)^{(n-p)} .
$$

Let $l=l(p, n)$ be the first positive integer such that $1 / l<1-p / n$. Then from (6.9) observe that

$$
\begin{aligned}
\left(m_{1} \cdots m_{j}\right)^{n}\left(s_{j}\right)^{(n-p)} & =\left(m_{1} \cdots m_{j-1}\right)^{n}\left(s_{j-1}\right)^{n / j}\left(s_{j}\right)^{n(1-p / n-1 / j)} \\
& \leq\left(m_{1} \cdots m_{j-1}\right)^{n}\left(s_{j-1}\right)^{n-p} \\
& \leq \cdots \leq\left(m_{1}, \ldots, m_{l}\right)^{n}\left(s_{l}\right)^{n-p}, \quad j \geq l .
\end{aligned}
$$

Using (6.10) it follows that

$$
\int_{D_{i}}\left|\nabla u_{j}\right|^{p} d x \leq c\left(m_{1} \cdots m_{l}\right)^{n}\left(s_{l}\right)^{(n-p)}, \quad j \geq l .
$$

Now (6.8) implies that the distance between successive cubes in $K_{j}$ is at least $\left(2 m_{j}\right)^{-1} s_{j-1}$, when $j \geq 10$. Using this fact we deduce for $Q \in L_{j-1}, d=d(\cdot, \partial D)$, and $\tau=\left(2 m_{j}\right)^{-1} s_{j-1}$

$$
\begin{aligned}
\int_{Q}\left(\frac{u_{j}}{d}\right)^{p} d x & \geq c\left(m_{j}\right)^{n} \int_{10 s_{j}}^{\tau} \rho^{n-p-1} d \rho \geq c\left(m_{j}\right)^{n} \tau^{n-p} \\
& \geq c\left(m_{j}\right)^{p}\left(s_{j-1}\right)^{n-p}
\end{aligned}
$$


We now choose

$$
m_{j}=\left[(j+1)\left(s_{j-1}\right)^{1-n / p}\right]
$$

where $[x]$ denotes the greatest integer $\leq x$. Since $s_{j}=\left(s_{j-1}\right)\left(m_{j}\right)^{-j}$, the sequence $\left(m_{j}\right)_{1}^{\infty}$ is well defined by induction. Also from (6.11), (6.12), we conclude

$$
\int_{D}\left(u_{j} / d\right)^{p} d x \rightarrow+\infty \quad \text { as } j \rightarrow \infty
$$

while $\int\left|\nabla u_{j}\right|^{p} d x$ remains bounded independently of $j$. Finally it is easily shown that $F$ has Hausdorff dimension zero. Simply take the cubes in $L_{j}, j=1,2, \ldots$, as coverings and use (6.9). We omit the details. The proof of Theorem 3 is now complete.

7. Proof of Theorem 4. Let $E$ be a compact subset of $B(0,1)$ and $\alpha$ fixed, $0<\alpha<n / 2$. Suppose that $E$ is $(\alpha, 2)$ locally uniformly fat for some $\lambda, r_{0}>0$. Then clearly,

$$
r^{(2 \alpha-n)} R_{\alpha, p}[E \cap B(x, r)] \geq c \lambda r_{0}^{(n-2 \alpha)}=\tilde{\lambda},
$$

for $0<r<2$ and every $x \in E$. Let $\left(x_{i}\right)_{1}^{m}$ be a sequence of $m$ Fekete points for $E$. We assume, as we may, that

$$
\min _{i \neq j}\left|x_{i}-x_{j}\right|=\left|x_{1}-x_{2}\right|
$$

Let

$$
P(x)=\frac{1}{(m-1)} \sum_{i=2}^{m}\left|x-x_{i}\right|^{(2 \alpha-n)}, \quad x \in \mathbf{R}^{n} .
$$

From the minimizing property of Fekete points mentioned in $\S 1$, it is easily shown that

$$
P\left(x_{1}\right) \leq P(x), \quad x \in E .
$$

Put $u=P\left(x_{1}\right)-P$. We claim that

$$
M(r)=M\left(r, u, x_{1}\right) \leq c P\left(x_{1}\right) r^{\sigma}, \quad 0<r<1,
$$

where $c, \sigma$, are positive constants $(0<\sigma<1)$, depending only on $r_{0}, \lambda, n$, and $\alpha$. To prove this claim let $\mu$ be the measure with mass $1 /(m-1)$ at $x_{j}, 2 \leq i \leq m-1$, and observe that $P=I_{2 \alpha} * \mu$. We now repeat the argument in $\S \S 2$ and 3 using the fact that $I_{\alpha} * I_{\alpha}=\gamma I_{2 \alpha}$ for some $\gamma>0$. The argument is unchanged up to the point in $\S \S 2$ and 3 where we obtained

$$
M(s, h) \leq c I_{\alpha} *\left(I_{\alpha} * \mu_{1}\right)=c I_{2 \alpha} * \mu_{1}
$$

$(\alpha, 2)$ q.e. on $E \cap B\left(x_{1}, s_{1} / 8\right)$. Let $\nu$ be capacitary measure for $E \cap B\left(x_{1}, s_{1} / 8\right)$ and $H=\gamma I_{2 \alpha} * \nu$ the corresponding equilibrium potential. Then from (7.4), the Tonelli Theorem, and the fact that $H$ is bounded (see $[2,9]$ ), we obtain

$$
\begin{gathered}
M(s, h) R_{\alpha, 2}\left[E \cap B\left(x_{1}, s_{1} / 8\right)\right]=M(s, h) \nu\left[E \cap B\left(x_{1}, s_{1} / 8\right)\right] \\
\leq c \int\left(I_{2 \alpha} * \mu_{1}\right) d \nu=c \int\left(I_{2 \alpha} * \nu\right) d \mu_{1} \leq c \mu_{1}\left(\mathbf{R}^{n}\right) .
\end{gathered}
$$


The above argument was used in place of the argument in $\S \S 2$ and 3 because $I_{2 \alpha} * \mu_{1}$ need not be bounded by $M(s, h)$ on supp $\mu_{1}$. The rest of the argument is unchanged from the argument in $\S \S 2$ and 3. Hence (7.3) is true. Let $r=4\left|x_{1}-x_{2}\right|$ and put

$$
h(x)=P(x)-\frac{1}{(m-1)}\left|x-x_{2}\right|^{(2 \alpha-n)}, \quad x \in \mathbf{R}^{n} .
$$

To prove Theorem 4 we consider two cases. If $\alpha \geq 1$ let $r=4\left|x_{2}-x_{1}\right|$ and observe from (7.3) and superharmonicity of $h$ that

$$
\begin{aligned}
P\left(x_{1}\right) & \leq \min _{x \in \partial B\left(x_{1}, r\right)} P+c P\left(x_{1}\right) r^{\sigma} \\
& \leq \frac{1}{(m-1)} 2^{(n-2 \alpha)} r^{(2 \alpha-n)}+\min _{x \in \partial B\left(x_{1}, r\right)} h+c P\left(x_{1}\right) r^{\sigma} \\
& \leq \frac{1}{(m-1)} 2^{(n-2 \alpha)} r^{(2 \alpha-n)}+h\left(x_{1}\right)+c P\left(x_{1}\right) r^{\sigma} .
\end{aligned}
$$

Since

$$
h\left(x_{1}\right)=P\left(x_{1}\right)-\frac{1}{(m-1)}\left|x_{2}-x_{1}\right|^{(2 \alpha-n)}
$$

and $r=4\left|x_{1}-x_{2}\right|,(7.5)$ implies

$$
\frac{1}{(m-1)}\left|x_{2}-x_{1}\right|^{(2 \alpha-n)} \leq c\left|x_{2}-x_{1}\right|^{\sigma} P\left(x_{1}\right),
$$

or equivalently that

$$
\left|x_{2}-x_{1}\right| \geq\left[\frac{1}{(m-1) P\left(x_{1}\right)}\right]^{1 /(n-2 \alpha+\sigma)} .
$$

If $0<\alpha<1$ let $0<s<\frac{1}{2}$ and replace $\alpha$ by $2 \alpha$ in the definition of $Q_{s}$ following (3.1). Then $h$ is $2 \alpha$ superharmonic in $\mathbf{R}^{n}$. Hence

$$
\int_{\mathbf{R}^{n}-B\left(x_{1}, s\right)} Q_{s}(x, y) h(y) d y \leq h(x),
$$

whenever $x \in B\left(x_{1}, s\right)$. Now

$$
\begin{aligned}
1 & =\int_{\mathbf{R}^{n}-B\left(x_{1}, s\right)} Q_{s}(x, y) d y=\int_{\mathbf{R}^{n}-B\left(x_{1}, s^{1 / 2}\right)} \cdots+\int_{B\left(x_{1}, s^{1 / 2}\right)-B\left(x_{1}, s\right)} \cdots \\
& \leq c s^{2 \alpha} \int_{s^{1 / 2}}^{\infty} t^{-(1+2 \alpha)} d t+\int_{B\left(x_{1}, s^{1 / 2}\right)-B\left(x_{1}, s\right)} \cdots \\
& \leq c s^{\alpha}+\int_{B\left(x_{1}, s^{1 / 2}\right)-B\left(x_{1}, s\right)} Q_{s}(x, y) d y .
\end{aligned}
$$

Choose $s_{0}$ so that $c s_{0}^{\alpha}=\frac{1}{2}$, where $c$ is the constant in the last inequality. Given $s$, $0<s<s_{0}$, it follows from (7.7), (7.8), that there exists $r, s<r<s^{1 / 2}$, such that

$$
\min _{x \in \partial B\left(x_{1}, r\right)} h(x) \leq \frac{1}{\left(1-c s^{\alpha}\right)} h\left(x_{1}\right) \leq\left(1+2 c s^{\alpha}\right) h\left(x_{1}\right) .
$$

If $s=4\left|x_{1}-x_{2}\right|$ we can use the above inequality and repeat the argument used in (7.5) to obtain

$$
\frac{1}{(m-1)}\left|x_{1}-x_{2}\right|^{(2 \alpha-n)} \leq c\left(s^{\alpha}+r^{\sigma}\right) P\left(x_{1}\right) \leq c s^{\beta} P\left(x_{1}\right),
$$


where $\beta=\min (\alpha, \sigma / 2)$. From the above discussion we see that either $4\left|x_{1}-x_{2}\right| \geq$ $s_{0}$, in which case there is nothing to prove or (7.9) holds with $s=4\left|x_{1}-x_{2}\right|$. For this choice of $s,(7.9)$ yields

$$
\left|x_{1}-x_{2}\right| \geq c\left[1 / m P\left(x_{1}\right)\right]^{1 /(n-2 \alpha+\beta)},
$$

$m=1,2, \ldots$. To complete the proof of Theorem 4 it remains to show that

$$
P\left(x_{1}\right) \leq c .
$$

To do this let $\tau$ be capacitary measure for $E$. Then from (7.2), the fact that $I_{2 \alpha} * \tau \leq c$, and the Tonelli Theorem, we obtain

$$
\begin{aligned}
P\left(x_{1}\right) R_{\alpha, 2}(E) & \leq \int P d \tau=\int\left(I_{2 \alpha} * \tau\right) d \mu \\
& \leq c \mu\left(\mathbf{R}^{n}\right)=c .
\end{aligned}
$$

This inequality and (7.1) clearly imply (7.11). From (7.11), (7.10), and (7.6) we conclude that Theorem 4 is true.

As mentioned in $\S 1$, Theorem 4 has an analogue when $\alpha=n / 2$. In this case $\left(x_{i}\right)_{1}^{m}$ is said to be a sequence of $m$ Fekete points of $E$ provided it minimizes

$$
-\sum_{i \neq j} \log \left|y_{i}-y_{j}\right|
$$

over all sequences $\left(y_{i}\right)_{1}^{m}$ consisting of $m$ points in $E$. Suppose $E$ is $(n / 2,2)$ locally uniformly fat and

$$
\min _{i \neq j}\left|x_{i}-x_{j}\right|=\left|x_{1}-x_{2}\right| \text {. }
$$

As previously let

$$
P(x)=-\frac{1}{(m-1)} \sum_{i=2}^{m} \log \left|x-x_{i}\right|, \quad x \in \mathbf{R}^{n} .
$$

Again it can be shown for $u=P\left(x_{1}\right)-P$ that

$$
M(r) \leq c P\left(x_{1}\right) r^{\sigma}
$$

for some $\sigma>0$. The argument is similar to the argument following (7.3), except that now the results in $\S 4$ are used instead of those in $\S \S 2$ and 3. Also, one needs to use the fact that if $c>0$ is large enough, then

$$
\log \left[\frac{1}{(c|x|)}\right] \leq J_{n / 2} * J_{n / 2}(x) \leq \log \left[\frac{c}{|x|}\right], \quad x \in B(0,2) .
$$

We leave the details to the reader. Using (7.12) the argument for $\alpha \geq 1$ can be repeated to get

$$
\min _{i \neq j}\left|x_{i}-x_{j}\right| \geq c m^{-\sigma} \text {. }
$$

\section{REFERENCES}

1. D. Adams, Traces of potentials. II, Indiana Univ. Math. J. 22 (1973), 907-918.

2. D. R. Adams and N. G. Meyers, Thinness and Wiener criteria for nonlinear potentials, Indiana Univ. Math. J. 22 (1972), 169-197. 
3. D. R. Adams and L. Hedberg, Inclusion relations among finetopologies in nonlinear potential theory, Indiana Univ. Math. J. 33 (1984), 117-126.

4. D. R. Adams and N. G. Meyers, An equivalence of two definitions of capacity, Proc. Amer. Math. Soc. 37 (1973), 529-534.

5. __ Thinness and Wiener criteria for nonlinear potentials, Indiana Univ. Math. J. 22 (1972), 169-197.

6. A. Ancona, On strong barriers and an inequality of Hardy for domains in $\mathbf{R}^{n}$, J. London Math. Soc. (2) 34 (1986), 274-290.

7. L. Carleson, An interpolation problem for bounded analytic functions, Amer. J. Math. 80 (1958), 921-930.

8. B. Dahlberg, On the distribution of Fekete points, Duke Math. J. 45 (1978), 537-542.

9. V. Havin and V. Maz'ja, Nonlinear potential theory, Uspekhi Mat. Nauk 27 (1972), 67-138.

10. W. Hayman and C. Pommerenke, On analytic functions of bounded mean oscillation, Bull. London Math. Soc. 10 (1978), 219-224.

11. L. Hörmander, $L^{p}$ estimates for (pluri) subharmonic functions, Math. Scand. 20 (1967), 65-78.

12. N. Landkof, Foundations of modern potential theory, Springer-Verlag, 1972.

13. J. Lewis, Some applications of Riesz capacities, J. Computer Analysis (to appear).

14. N. G. Meyers, A theory of capacities for potentials of functions in Lebesgue classes, Math. Scand. 26 (1970), 255-292.

15. C. B. Morrey, Multiple integrals in the calculus of variations, Springer-Verlag, 1966.

16. C. Pommerenke, Über die Verteilung der Fekete Punkte. II, Math. Ann. 179 (1969), 212-218.

17. P. Sjögren, On the regularity of the distribution of the Fekete points of a compact surface in $\mathbf{R}^{n}$, Ark. Mat. 11 (1973), 148-151.

18. D. Stegenga, A geometric condition which implies BMOA, Proc. Sympos. Pure Math., vol. 35, part 1, Amer. Math. Soc., Providence, R.I., 1979, pp. 427-430.

19. E. Stein, Singular integrals and differentiability properties of functions, Princeton Univ. Press, Princeton, N.J., 1970.

Department of Mathematics, University of Kentucky, Lexington, KenTUCKY 40506 